\title{
Giant cell tumour of tendon sheath (localised nodular tenosynovitis): clinicopathological features of 71 cases
}

\author{
H Monaghan, D M Salter, A Al-Nafussi
}

\begin{abstract}
Aims/Background-Giant cell tumour of the tendon sheath (GCTTS) is regarded as the most common neoplasm of the hand that can recur after excision. The objective of this study was to review a series of cases in our department and to determine any clinical or pathological features that might predict the likelihood of recurrence.

Methods-Clinical data, obtained from pathology request forms and in patient notes, along with the gross and microscopic appearances of 71 cases of GCTTS were evaluated.

Results-Clinical features and pathological features identified were similar to those of previous studies. In comparison with previous studies a higher mitotic count (range, 1-21 mitoses/10 high power fields (HPF); mean, 5/10 HPF) was noted in all cases, irrespective of recurrence and numerous apoptotic bodies (up to 30/10 HPF), mainly formed from osteoclast-like giant cells, were present.

Conclusions-GCTTS is a relatively rare soft tissue tumour of uncertain histiogenesis. Mitotic and apoptotic figures are a common feature and do not indicate clinical behaviour. Complete local excision is the treatment of choice.

(f Clin Pathol 2001;54:404-407)
\end{abstract}

Keywords: giant cell tumour tendon sheath; apoptosis; osteoclasts

Giant cell tumour of the tendon sheath (GCTTS) is a benign lesion, most commonly occurring in hands and feet, but also found around the ankle and knee joints. ${ }^{12}$ GCTTS affects individuals between the age of 30 to 50 years and is more often seen in women than in men. ${ }^{2}$ The nature and cause of GCTTS are unclear. It has been considered to be an inflammatory process arising as a consequence of chronic antigenic stimulation; a reactive proliferation developing from the synovial lining of the tendon sheath and joint ${ }^{3}$; or a lesion of monocyte/macrophage derivation. ${ }^{4}$ Data from previous studies have shown a $50 \%$ history of trauma and multifocality, and similar lesions can be induced experimentally after extra articular injection of blood in experimental animals. ${ }^{4}$ Recent studies demonstrating cytogenetic abnormality in the form of trisomy 7 and autonomous growth, in addition to the clinical features of local recurrence and case reports of metastatic GCTTS, raised the possibility that it is a cancer. ${ }^{5}{ }^{6}$ However, Vogrincic et al used a polymerase chain reaction (PCR) based assay for methylation of the $\mathrm{X}$ linked human androgen receptor (HUMARA) in female subjects to demonstrate that GCTTS is a polyclonal proliferation. They argued that if one accepted that a population of cells forming a tumorous mass must show clonality to be classified as a neoplasm, then GCTTS is either a reactive or hyperplastic process. ${ }^{7}$

A high mitotic rate is thought to be indicative of local recurrence, ${ }^{89}$ but there is debate as to how many mitoses are required. Our study was performed to ascertain whether mitotic figures were associated with recurrence and, if so, the numbers required.

\section{Materials and methods}

The department of pathology, Edinburgh University Medical School serves a population of 400000 . We searched the departmental surgical files between the years 1990-7 for cases reported as GCTTS or localised nodular tenosynovitis. We did not include cases of diffuse villoglandular tenosynovitis. The clinical data were obtained from request forms and in patient notes and evaluated together with the gross and microscopic appearances of the lesions. Haematoxylin and eosin stained sections were retrieved and examined for the following features: growth patterns, presence or absence of capsule, and its status and cellular constituents. The mitotic and apoptotic counts were assessed by randomly counting 10 different high power microscopic fields $(\times 400$ magnification; field size of $0.152 \mathrm{~mm}^{2}$ ) and were recorded as the number of mitotic or apoptotic figures/10 high power fields (HPF). Apoptotic figures accepted had a minimum size of $0.1 \mathrm{~mm}$.

\section{Results}

\section{CLINICAL FINDINGS}

We found a total of 71 patients within a period of eight years - about eight each year - and an incidence of $1 / 50000$. Of the 71 patients, 44 were women and 27 men, a female to male ratio of $1.6: 1$. The ages of the patients at presentation ranged from 6 to 71 years, with a mode in the 4 th decade and a mean age of 46.3 years. Follow up ranged from one to eight years.

The hands were involved in 66 of 71 patients, the feet in three patients, and tissues around the knee in two patients. The most common sites in the hands were the fingers (59
Accepted for publication 20 September 2000 


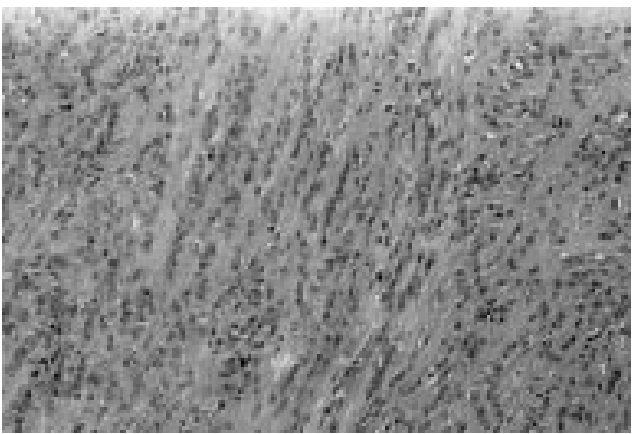

Figure 1 "Single file" pattern of mononuclear cells.

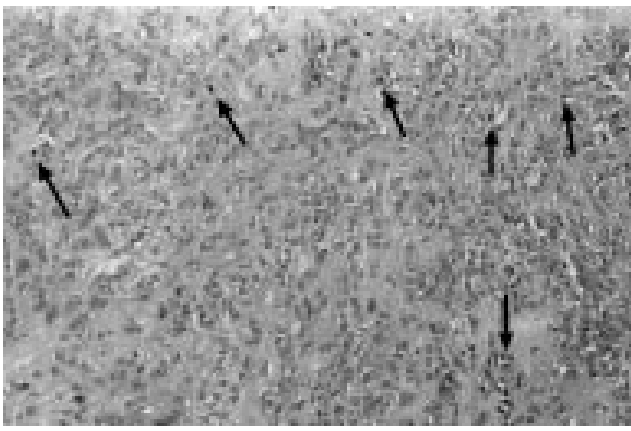

Figure 2 Mitotic figures (arrowed).

of 66 patients), the palm (six patients), and the wrist (one patient). In 51 of 59 patients the finger involved was specified on request forms. The index, middle finger, and the thumb were more commonly involved than the ring and the little finger. The lesion occurred more commonly on the fingers of the right hand ( 37 patients) than those on the left hand (23 patients).

The size of the lesions varied between $0.5 \mathrm{~cm}$ and $4.5 \mathrm{~cm}$ (mean, $1.75 \mathrm{~cm}$ ). The most common presentation was as a painless swelling (93\% of cases). In only three of 71 patients was a provisional clinical diagnosis of GCTTS made. In the remaining patients a diagnosis of ganglion or epidermoid cyst was offered.

The duration of the lesion before treatment varied between six months and five years and a history of antecedent trauma was noted in one patient. All cases were treated by local excision, removing the lesion with a minimal capsular margin of $1 \mathrm{~mm}$ of tissue. Tumour recurrence was seen in three patients; all recurrences occurred just after one year of the original excision. These were re-excised with no further recurrences.

\section{PATHOLOGICAL FINDINGS}

All tumours were well circumscribed, lobulated, or multinodular. A fibrous capsule of varying thickness was found in all patients. The cellular infiltrate was variably seen within the capsule as well as in the tissue immediately around the capsule. Varying degrees of hyalinisation were seen in almost all patients. In some patients, the hyalinisation was keloidal in type and occasionally had an osteoid-like appearance. Haemosiderin was identified in almost every patient.

The cellular constituents comprised four cell types, namely: macrophage-like mononuclear

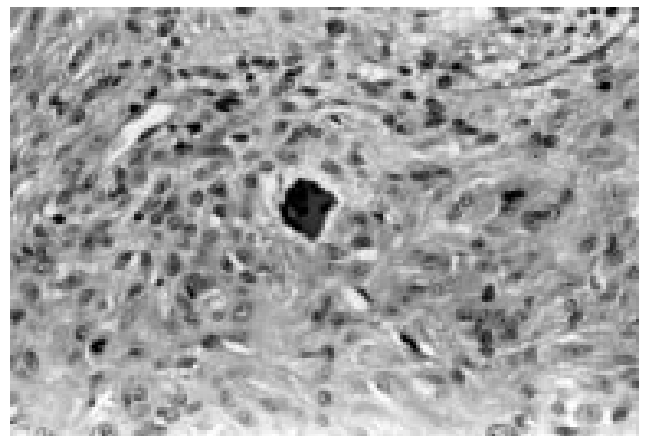

Figure 3 Apoptotic bodies forming from osteoclast-like giant cells.

cells, epithelioid histiocyte-like cells, osteoclast-like giant cells, and xanthomatous cells. The macrophage-like mononuclear cells formed most of the background cells. These had folded kidney shaped or "coffee bean", grooved oval, or occasionally spindle shaped nuclei with very small or no nucleoli. The cytoplasm was indiscernible; therefore, the nuclei appeared to blend into the background stroma. Usually, these cells were evenly distributed within the lesion, but sometimes they were arranged in rows or single files in a pattern reminiscent of invasive lobular carcinoma of the breast (fig 1). In one patient, the cells were arranged in a palisade around keloidal hyalinisation.

The mononucleated or binucleated epithelioid histiocyte-like cells had abundant glassy cytoplasm with round nuclei and prominent nucleoli. Although the number of these cells varied in different lesions their presence was ubiquitous. These cells were often seen at the periphery and appeared to blend with the cytoplasm of the third cell population-the osteoclast-like giant cells. The latter were seen in varying numbers, arranged in clusters, throughout the lesion, but in almost every case. The nuclei of the osteoclast-like giant cells were of both types; that is, folded or grooved with no discernible nucleoli and round with prominent nucleoli. The xanthomatous cells were variable in number and tended to aggregate focally. Their nuclei were identical to those of the macrophage-like cells in the remainder of the lesion. Touton-type giant cells were also seen in some cases. Occasional lymphocytes were seen within the lesions, frequently in aggregates at the periphery.

Mitotic figures were seen in every patient, ranging from 1 to $21 / 10 \mathrm{HPF}$, with an average of 5/10 HPF (fig 2). No atypical mitoses were identified. Apoptotic bodies were identified in almost every lesion, with up to $30 / 10 \mathrm{HPF}$. Numbers of apoptotic bodies were less in the hyalinising type (more than $50 \%$ of the lesion replaced with hyaline) rather than the cellular type (more than $75 \%$ cellular) of GCTTS (18/10 HPF $v 35 / 10 \mathrm{HPF})$. In most cases, the apoptotic bodies appeared to be derived from osteoclast-like giant cells, in that they clearly originated from multinucleate cells, and were found in areas with numerous osteoclast-like giant cells (fig 3). The three cases in which recurrence occurred had no unusual features, 
in particular the mitotic and apoptotic counts were not increased (with mitotic counts of 5, 11 , and $12 / 10 \mathrm{HPF}$ and apoptotic counts of 8 , 13 , and $18 / 10 \mathrm{HPF}$ ). There was no correlation between mitotic and apoptotic counts. Few of the patients underwent $x$ ray examination, but those who did showed no evidence of intraosseous invasion.

\section{Discussion}

We found an incidence of eight cases each year, which is slightly less than the incidence of 10.07 noted by Ushijima et al in Japan. ${ }^{1}$ Our mean age of presentation was 46.3 years, with a peak in the 4 th decade, in keeping with the peak ages of the $3 \mathrm{rd}$ and 4 th decades found in previous studies. ${ }^{110}$ Ninety three per cent of our patients had presented with a painless swelling, compared with $80 \%$ seen in other studies. ${ }^{10}$ This was more often on one of the fingers of the right hand. Most of the lesions were in the first three fingers of the right hand, raising the possibility of minor repetitive trauma as an aetiological factor. However, only one patient had a definite history of antecedent trauma, which is considerably less than previous studies, in which the incidence ranged from $10 \%$ to $50 \% .^{11}$ Fourteen per cent of lesions were present in small (digit) joints rather than large joints. This distribution was similar to that noted by Ushijima et al $(8.28 \%)$.

All the lesions in our patients with GCTTS were present adjacent to joints; however, it has been reported to occur at other sites, such as the upper back, where the tumour was attached to the periosteum of the seventh posterior intercostal space. ${ }^{12}$

The gross features of the lesions in our study are remarkably similar to previous studies, with a mean size of $1.75 \mathrm{~cm}$ and a range in size of 0.5 to $4.5 \mathrm{~cm}$ compared with a range of 0.5 to $4.0 \mathrm{~cm}$ noted by Enzinger and Weiss. ${ }^{5}$ Their lobulated nature is also a consistent feature.

Intraosseous invasion was not present in our cases; however, this may be explained in part by the fact that most of our cases occurred in small joints and were of localised type rather than diffuse type. Intraosseous invasion has been mainly documented in large joints with diffuse GCTTS. ${ }^{10}$ Cortical erosion of bone has also been described previously, mainly in large joints.

The cell types described are also the same as those described in previous studies, namely: histiocyte-like cells, macrophage-mononuclear cells, osteoclast giant cells, and xanthomatous cells with variable numbers of Touton-type giant cells and lymphocytes.

Histology has confirmed the presence of mitoses. However, our study shows a higher number than previously described and the new observation of apoptotic bodies has been made. We found a mean count of 5 mitoses/10 $\mathrm{HPF}$ (range, 1-21/10 HPF) and mitotic figures were found in all lesions. Wright et al found mitoses in only $50 \%$ of lesions ${ }^{9}$ and Rao and Vigorita found three or more mitotic figures in each $10 \mathrm{HPF}$ in over $10 \%$ of their cases. ${ }^{8}$ Wright et al noted that recurrences occurred in highly cellular lesions with an increased number of mitoses, and Rao and Vigorita also suggested that the finding of three or more mitotic figures in each $10 \mathrm{HPF}$ might indicate an actively growing lesion, which was more likely to recur. Recent studies have also suggested that nm23 (a gene present in normal cells that is responsible for infiltration) can be used as a prognostic marker for the risk of recurrence. ${ }^{13}$ However, it seems probable that our lower rate of recurrence might be the result of adequate initial local excision (with resection margins being $>1 \mathrm{~mm}$ ), rather than the surgical technique of performing simple enucleations, thus leaving cells outside the capsule.

As suggested by the name, osteoclast-like giant cells are common in GCTTS. These were indistinguishable from other osteoclasts but in addition showed considerable numbers of apoptotic figures. The reasons for the accumulation of osteoclast-like giant cells in GCTTS is not clear, but numbers present will depend on recruitment and lifespan. Continued production of osteoclasts appears to occur as a result of the local production and activity of cytokines, including transforming growth factor $\beta 1$, macrophage colony stimulating factor, and parathyroid hormone related peptide, with mononuclear cells fusing to form giant cells with osteoclast morphology and phenotype. ${ }^{14} \mathrm{~A}$ high rate of osteoclast apoptosis might suggest lack of survival factors, such as interleukin 1, or the action of cytokines to induce apoptosis through interaction with tumour necrosis factor related apoptosis inducing ligand (TRAIL) receptors. ${ }^{14}$

The differential diagnosis of GCCTS includes giant cell tumour of soft tissue and giant cell tumour of bone. These tumours may be differentiated microscopically from GCTTS by the presence of necrosis, metaplastic bone formation, and aneurysmal bone cyst-like areas and their typical location of back, thigh, and shoulder. ${ }^{15}{ }^{16}$ Fibroma of tendon sheath and extraskeletal osteosarcoma should also be considered. However, the mixed cell population of GCTTS should distinguish it from the former and the presence of anaplasia, malignant spindle cell stroma, numerous atypical mitoses, and bone formation from the latter. The high mitotic count and single file pattern of mononuclear cells would be highly suspicious of metastatic lobular carcinoma of the breast in a small biopsy; however, the overall pattern of GCTTS should exclude this as a differential diagnosis.

In conclusion, GCTTS is a relatively rare soft tissue tumour of uncertain histiogenesis. Complete local excision is the treatment of choice. Mitotic and apoptotic figures are associated features but do not predict clinical behaviour.

1 Ushijima M, Hashimoto $H$, Tsuneyoshi $M$, et al. Giant cell tumour of the tendon sheath (nodular tenosynovitis). A study of 207 cases to compare the large joint group with the common digit group. Cancer 1986;57:875-84. 2 Jones FE, Soule EH, Coventry MB. Fibrous histiocytoma of synovium (giant cell tumour of tendon sheath, pigmented

3 Cavaliere A, Sidoni A, Bucciarelli E. Giant cell tumour of tendon sheath: immunohistochemical study of 20 cases. Tumori 1997;83:841-6. 
4 Geweiler JA, Wilson JW. Diffuse biarticular pigmented villonodular synovitis. Radiology 1969;93:845

5 Enzinger FM, WLis SW. Soft tissue tumo Mosby, 1982:736-55.

6 Castens HP, Howell RS. Malignant giant cell tumour of tendon sheath. Virchows Arch A Pathol Anat Histol 1979:382:237-43.

7 Vogrincic GS, O'Connell JX, Gilks CB. Giant cell tumour of tendon sheath is a polyclonal cellular proliferation. Hum Pathol 1997;28:815-19.

8 Rao AS, Vigorita VJ. Pigmented villonodular synovitis (giant cell tumour of tendon sheath and synovial membrane): a review of eighty-one cases. 7 Bone foint Surg Am 1984;66:76-94.

Wright CJE. Benign giant cell synovioma. $\mathrm{Br} f$ Surg 1951;38:257

10 Bertoni F, Unni KK, Beabout JW, et al. Malignant giant cell tumour of the tendon sheaths and joints (malignant pigmented villonodular synovitis). Am f Surg Pathol 1997; 21:153-63.
11 Crosby EB, Inglis A, Bullough PG. Multiple joint Crosby EB, Inglis A, Bullough PG. Multiple joint
involvement with pigmented villonodular synovitis. Radiolinvolvement with pig

12 Thaxton L, AbuRahma AF, Chang H-H, et al. Localised giant cell tumour of tendon sheath of upper back. Surgery 1995;118:901-3.

13 Grover R, Grobbelaar AO, Richman PI, et al. Measurement of invasive potential provides an accurate prognostic marker for giant cell tumour of tendon sheath. F Hand Surg [Br] 1998;23:728-31.

14 Wyllie AH. Apoptosis: an overview. Br Med Bull 1997;53: 451.

15 O'Connell JX, Wehrli BM, Nielsen GP, et al. Giant cell tumours of soft tissue: a clinicopathologic study of 18 benign and malignant tumours. Am f Surg Pathol 2000;24: 386-95.

16 Oliveira AM, Dei Tos AP, Fletcher CDM, et al. Primary giant cell tumour of soft tissues: a study of 22 cases. $A m \mathcal{F}$ Surg Pathol 2000;24:248-56.

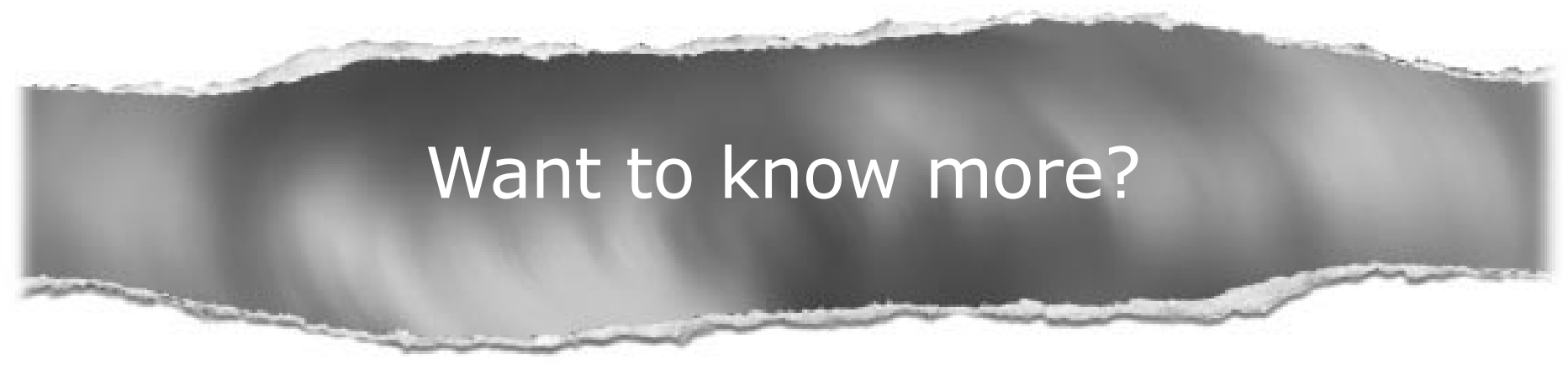

Data supplements

Limited space in printed journals means that interesting data and other material are often edited out of articles; however, limitless cyberspace means that we can include this information online. Look out for additional tables, references, illustrations.

\section{www.jclinpath.com}

